\title{
First experience with left atrial arrhythmia ablation using a bi- directional steerable transseptal sheath (Vizigo) visible in the CARTO system as a method to reduce fluoroscopy
}

\author{
Edward Koźluk ${ }^{1} \oplus$, Katarzyna Kojewska² $^{\circledR}$, Jarosław Hiczkiewicz²,3
}

${ }^{1}$ I Chair and Department of Cardiology, Medical University of Warsaw, Poland

${ }^{2}$ Clinical Department of Cardiology, Nowa Sól Multidisciplinary Hospital, Nowa Sól, Poland

${ }^{3}$ Collegium Medicum, University of Zielona Góra, Poland

\begin{abstract}
In this report we present ablations of complex left atrial arrhythmias in 3 male patients using the bi-directional steerable transseptal sheath (Visigo) which is visualizable by the 3D electro-anatomical system. Ablations of complex left atrial (LA) arrhythmias were performed in 3 patients. In the first 2 patients typical transseptal punctures were performed, followed by mapping with the LassoNav catheter and PVI (one patient also had isolation of the posterior segment). The last patient had a residual atrial septal leak, therefore ablation without fluoroscopy was attempted. An anatomical map of the right atrium was made. The ablation catheter and the Vizigo sheath were introduced into the LA through the leak in the septum. LA, pulmonary veins and 3 tachycardia loops were mapped. Lines were made in the roof of $L A$, in the mitral isthmus and within the atrial septum, restoring the sinus rhythm. Times of procedures/fluoroscopy were: $185,185,205 \mathrm{~min} / 5.5 ; 3.8$ and $0 \mathrm{~min}$. In the group of the last 10 previous ablations, these times were respectively: $209 \pm 48 \mathrm{~min} / 5,6 \pm 1,8 \mathrm{~min}$. We conclude that the Vizigo sheath reduces the risk of electrode and sheath dislocation into the right atrium and the need for fluoroscopic verification during maneuvers performed with the sheath. It is also a step towards simpler left atrial ablation without the use of fluoroscopy.
\end{abstract}

Keywords: atrial fibrillation ablation $\cdot$ pulmonary vein isolation $\cdot$ zero fluoroscopy $\cdot$ steerable sheath $\cdot$ electroanatomic mapping system

\section{Citation}

Koźluk E, Łojewska K, Hiczkiewicz J. First experience with left atrial arrhythmias ablation using visualizable by CARTO system bi-directional steerable transseptal sheath (Vizigo) as a method to reduce fluoroscopy. Eur J Transl Clin Med. 2020;3(2)18-21.

DOI: $10.31373 /$ ejtcm/131049

\section{Corresponding author:}

Edward Koźluk, PhD MD, I Chair and Department of Cardiology, Medical University of Warsaw

e-mail: elektrofizjologia@wum.edu.pl

No external funds.

Available online: www.ejtcm.gumed.edu.pl

Copyright $\circledast$ Medical University of Gdańsk

This is Open Access article distributed under the terms of the Creative Commons Attribution-ShareAlike 4.0 International.

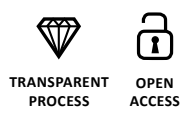


Introduction

In this report we present ablations of complex left atrial arrhythmias in 3 male patients using the bi-directional steerable transseptal sheath (Vizigo by Johnson \& Johnson, New Brunswick, USA) visualizable by the 3D electro-anatomical 3D system.

\section{Material and methods}

The first patient was a 70-year-old male with history of 2 pulmonary vein isolations (PVI) procedures, hypertension (HTN) and hyperthyroidism. The second patient was a 67-year-old male after PVI, with history of coronary artery disease and HTN. The third patient was a 71-year-old male with persistent left atrial flutter (LAFI) and history of PVI and HTN.

In the first 2 patients, short right atrium map was performed to create matrix for visualization of the Vizigo sheath (Figure 1A). Typical transseptal punctures were performed followed by mapping with the LassoNav catheter (Johnson \& Johnson) and PVI (in the second patient also isolation of the posterior segment). Ablation Index algorithm was used and the distance between neighboring ablation points was $<6 \mathrm{~mm}$. This strategy guarantees the continuity of the ablation line in the atria. The third patient had a residual atrial septal leak after previous procedure and was therefore attempted to ablate without fluoroscopy.

An anatomical map of the right atrium was made. On its basis, the Navistar ThermoCool SmartTouch SF ablation catheter (Johnson \& Johnson) and Vizigo sheath were introduced into the left atrium through the leak in the septum (Figure 1B,C,D,E, Fig.2). The ablation catheter was replaced with a multipoint PenthaRay mapping catheter dedicated for high-density mapping (Johnson \& Johnson) and an ablation electrode with a classic sheath was inserted through the same puncture (Fig.3). Left atrium with pulmonary veins and 3 tachycardia loops were mapped. Lines were made in the critical points of these loops: in the roof of the left atrium, in the mitral isthmus and within the atrial septum, restoring the sinus rhythm (final map - Figure 1D). Times of procedures and fluoroscopy were, respectively: 185, 185, 205 min / 5.5; 3.8 and Omin (dose area product, DAP: 76,4, 55,8 and $0 \mathrm{mGy}$ ). To explain the meaning of these values describing the procedure, we present the values of these parameters in the last 10 patients who underwent similar procedures without the use of a Vizigo sheath. These times were respectively: $209 \pm 48 \mathrm{~min} / 5,6 \pm 1,8 \mathrm{~min}$ (DAP $52,9 \pm 15,6 \mathrm{mGy})$. Procedural endpoints were achieved in all patients. There was no procedural complication noted.

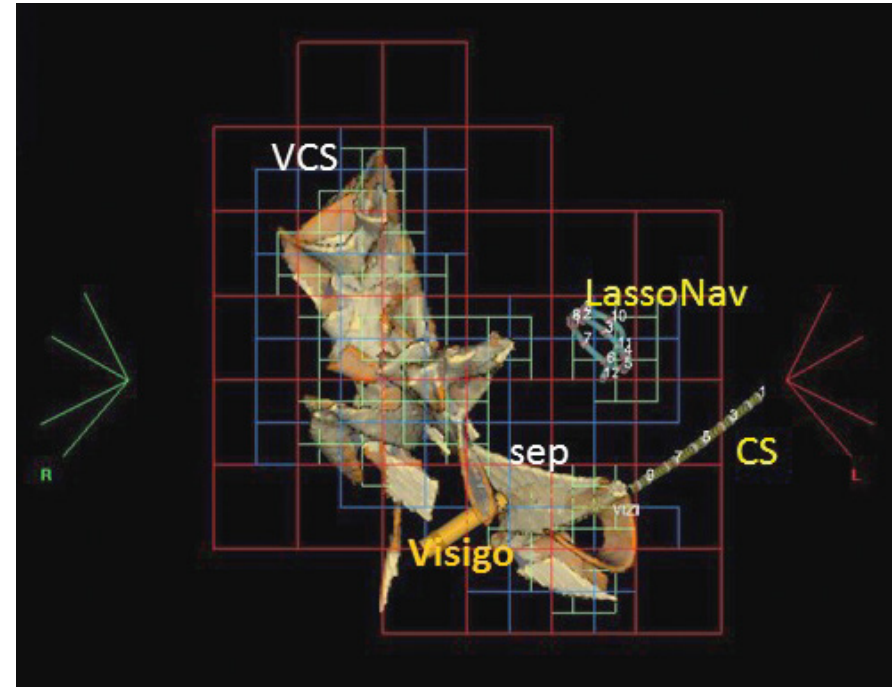

Fig.1A. AP projection. Matrix formed by Navistar SmartTouch ablation catheter and Lasso catheter (visible in the background as a colored grid) to accurately locate and visualize a steerable transseptal sheath. The fast-anatomical map of the upper part of the right atrium and the septum area is visible.

CS - the coronary sinus dekapolar catheter, LassoNav - Lasso Nav catheter in the left atrium, sep - interatrial septum, VCS - the superior vena cava, Vizigo - bi-directional steerable transseptal sheath Vizigo.

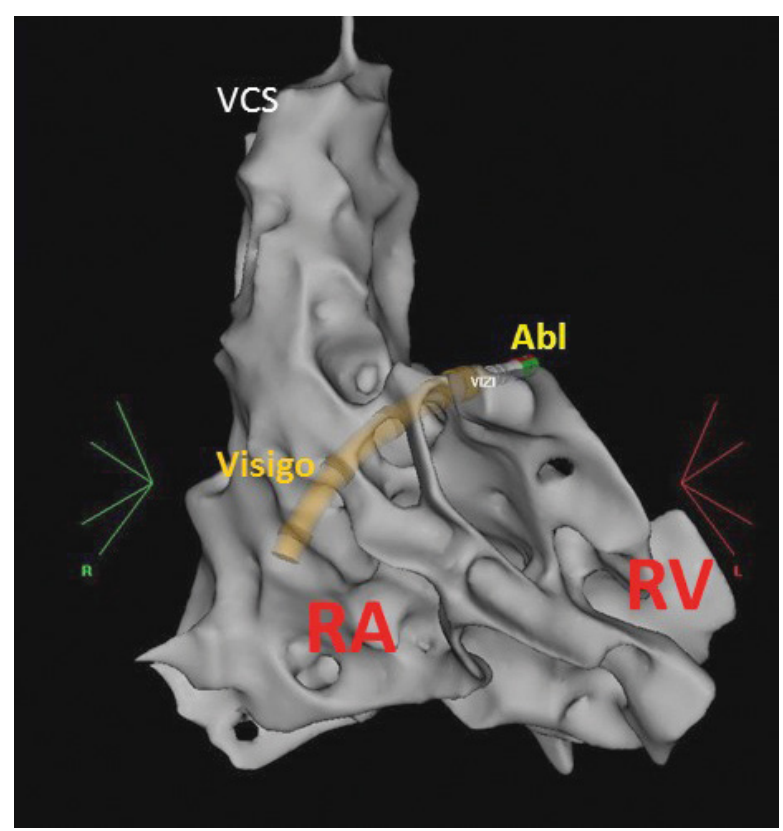

Fig. 1B. RAO projection of the fast-anatomical map of the right atrium. Vizigo sheath over the ablation catheter passing through the interatrial septum from the right to the left atrium. Abl-ablation catheter (Navistar SmartTouch) RA - the right atrium, RV - the right ventricle, VCS - the superior vena cava, Vizigo - bi-directional steerable transseptal sheath Vizigo 


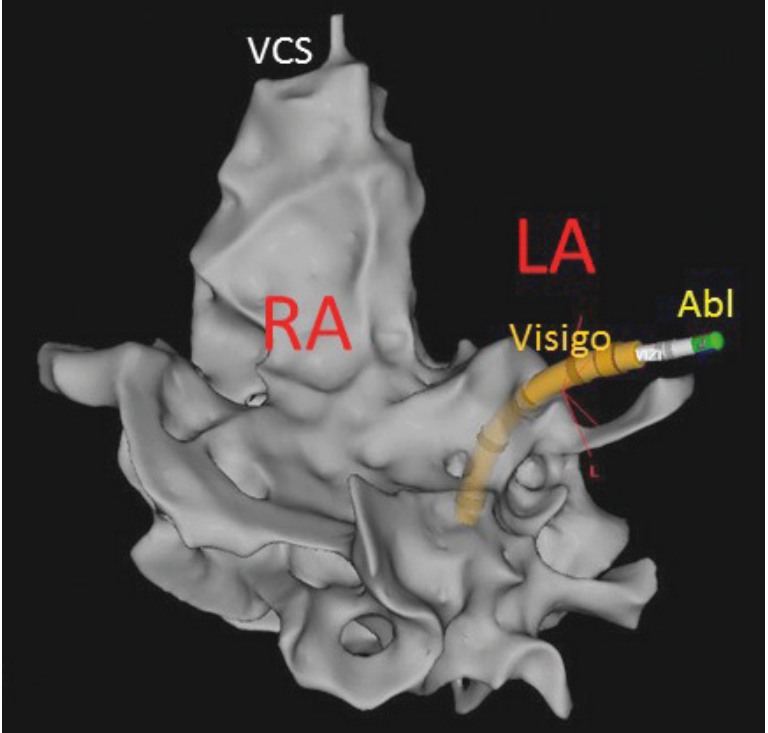

Fig. 1C. LAO projection of the fast-anatomical map of the right atrium. Vizigo sheath over the ablation catheter passing through the interatrial septum from the right to the left atrium. Abl - ablation catheter (Navistar SmartTouch) LA - the left atrium space (without the map at this level of procedure), RA - the right atrium, VCS - the superior vena cava, Vizigo - bi-directional steerable transseptal sheath Vizig

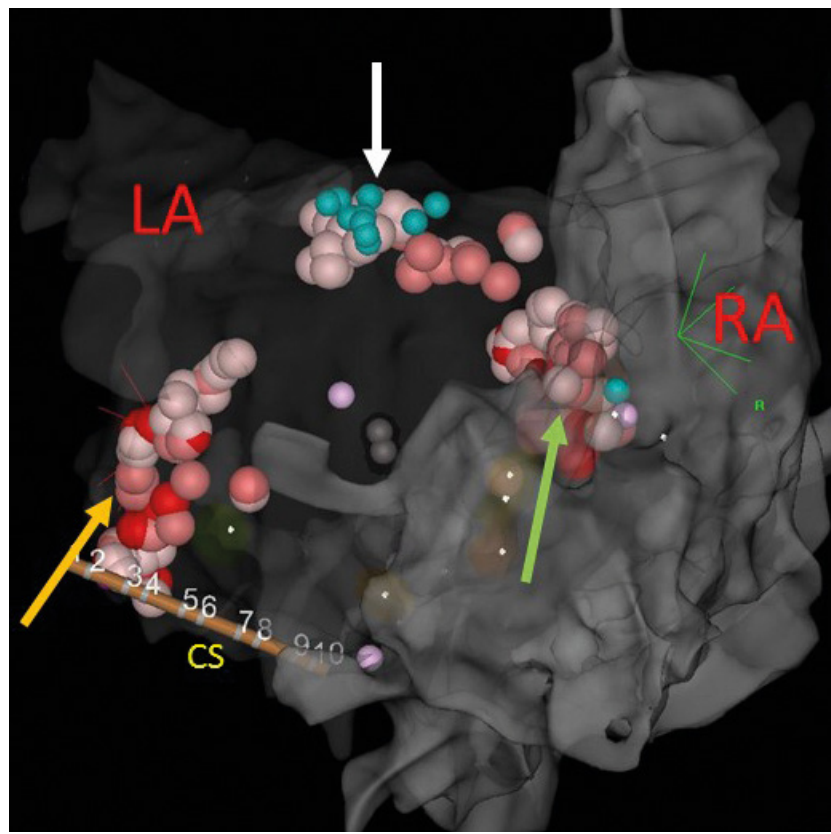

Fig. 1D. The final anatomical maps of the left (with transparency) and the right atrium after the procedure was finished. Modified posterior view. Small dots - places with fragmented potentials.

Larger blue dots - ablation points: white with ablation index 350-400, pink with ablation index 400-549, red with ablation index $>549$. White arrow - the line in the left atrial roof. Yellow arrow - the mitral line. Green arrow - the septal line.

CS - the coronary sinus decapolar catheter, LA - the left atrium, RA - the right atrium

\section{Discussion}

Due to the harmful side effects of ionizing radiation we paid attention to the radiological exposure of the patients and medical staff during these procedures. This resulted in the introduction of the ALARA concept, which recommends rational minimization of fluoroscopy [1]. The aim is to perform the procedure with zero fluoroscopy [2]. Literature on fluoroless ablation of atrial fibrillation describes the use of intracardiac or transesophageal echocardiography in combination with an electroanatomical system [3-4]. However, the use of the intracardiac echocardiography significantly increases costs and adds complexity due to the use of an additional vascular puncture and an additional catheter. Transesophageal echocardiography is a significant discomfort for the patient and by triggering his/her cough reflex may increase the risk complications. Therefore, the introduction of a transseptal sheath visualized by an electroanatomical system may simplify such procedures and potentially increase their safety.

\section{Conclusions}

Based on our initial 3 procedures, we conclude that the Vizigo sheath reduces the risk of electrode and sheath dislocation into the right atrium and the need for fluoroscopic verification during maneuvers performed with the sheath. It is also a step towards simpler left atrial ablation without the use of fluoroscopy.

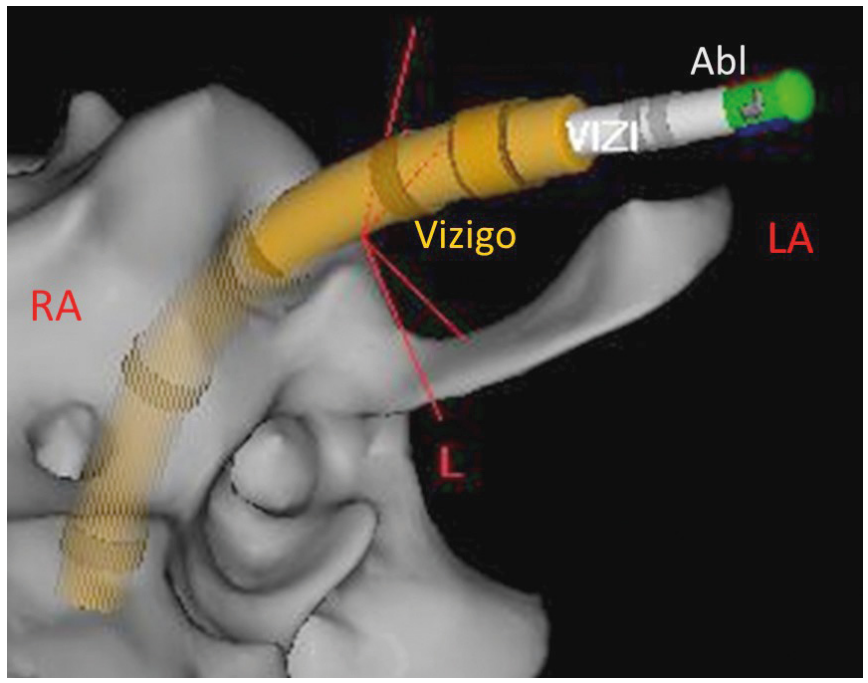

Fig. 1E. - enlarged region with Vizigo sheath (with ablation catheter inside) running through the interatrial septum.

Abl - ablation catheter (Navistar SmartTouch) LA - the left atrium space, RA - the right atrium, Vizigo - bi-directional steerable transseptal sheath Vizigo. 


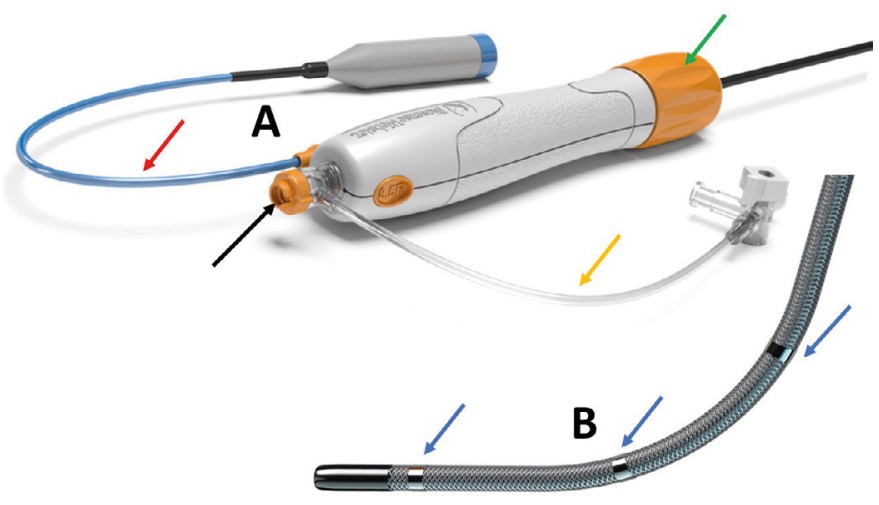

Fig. 2. Vizigo transseptal sheath.

A - Handle. Black arrow - lumen for catheter, red arrow - electrical cable connecting the sheath with the 3D system, yellow arrow - rinsing tube, green arrow - knob bending the sheath.

B - Distal steerable end with four distal electrodes (three of them are visible here) enable real-time steerable sheath visualization (blue arrows).
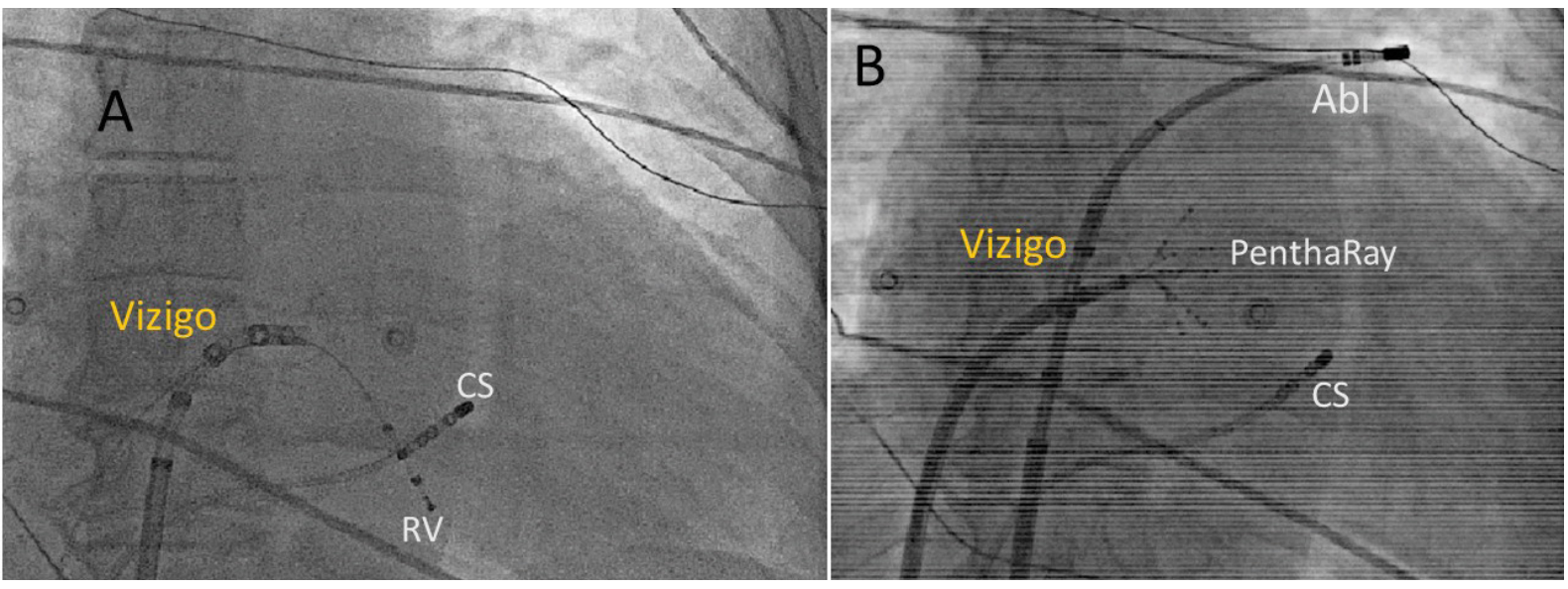

Fig. 3. Fluoroscopy picture presenting Vizigo sheath (example from another patient than presented in this report because our patient had ablation without fluoroscopy).

A - fluoro view with Vizigo catheter in the left atrium before introducing catheters there.

B - ablation Navistar catheter introduced via Vizigo sheath into the left superior pulmonary vein and PenthaRay catheter introduced via classical transseptal sheath.

Abl - ablation catheter (Navistar SmatTouh), LA - the left atrium space, RA - the right atrium, Vizigo - bi-directional steerable transseptal sheath Vizigo.

\section{References}

1. Hirshfeld JW, Balter S, Brinker JA, Kern MJ, Klein LW, Lindsay BD, et al. ACCF/AHA/HRS/SCAI Clinical Competence Statement on Physician Knowledge to Optimize Patient Safety and Image Quality in Fluoroscopically Guided Invasive Cardiovascular Procedures. Circulation [Internet]. 2005 Feb 1;111(4):511-32. Available from: https://doi.org/10.1161/01. CIR.0000157946.29224.5D

2. Koźluk E, Gawrysiak M, Piątkowska A, Lodziński P, Kiliszek M, Małkowska S, et al. Radiofrequency ablation without the use of fluoroscopy - in what kind of patients is it feasible? Arch Med Sci [Internet]. 2013/11/05. 2013 Oct 31;5(5):821-5. Available from: https://pubmed.ncbi.nlm.nih.gov/24273563

3. Žižek D, Antolič B, Prolič Kalinšek T, Štublar J, Kajdič N, Jelenc M, et al. Intracardiac echocardiography-guided transseptal puncture for fluoroless catheter ablation of left-sided tachycardias. J Interv Card Electrophysiol [Internet]. 2020 Aug 28; Available from: http://link.springer.com/10.1007/s10840-020-00858-z

4. O'Brien B, Balmforth DC, Hunter RJ, Schilling RJ. Fluoroscopy-free AF ablation using transesophageal echocardiography and electroanatomical mapping technology. J Interv Card Electrophysiol [Internet]. 2017 Dec 14;50(3):235-44. Available from: http://link.springer.com/10.1007/s10840-017-0288-9 